\title{
LAS FECHAS C-14 DEL CASTRO DE O ACHADIZO (BOIRO, A CORUÑA): PROBLEMÁTICA DE LA CALIBRACIÓN DE CONCHAS MARINAS
}

\author{
RADIOCARBON DATES OF THE IRON AGE \\ HILLFORT OF O ACHADIZO (BOIRO, A CORUÑA, \\ NW IBERIA): AN APPROACH TO THE DATING \\ OF MARINE SHELLS
}

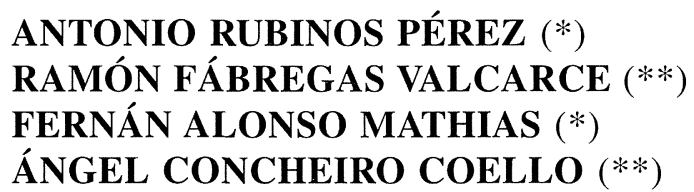

\section{RESUMEN}

La excavación del castro de OAchadizo (Boiro, A Coruña) puso al descubierto una serie de estructuras domésticas, así como varios basureros, encuadrables todos ellos dentro de la cultura castreña del Noroeste ibérico. El hecho de poder disponer de una gran cantidad de material orgánico procedente de los distintos niveles de habitación ha permitido llevar a cabo un programa sistemático de datación mediante el radiocarbono. Como resultado de los análisis radiométricos se deduce que la principal ocupación del yacimiento tuvo lugar entre los siglos VI al II cal BC. Por otra parte hemos cotejado las dataciones obtenidas sobre muestras con la misma procedencia estratigráfica, de origen terrestre (huesos y carbones) y oceánico (conchas), a fin de evaluar el efecto de reserva marina. El valor medio de la edad aparente de las conchas de O Achadizo (316 \pm 19$)$ es semejante al calculado para la costa portuguesa y subraya la necesidad de efectuar una corrección de las dataciones hechas sobre material orgánico de origen marino.

(*) Laboratorio de Geocronología. Instituto de Química-Física "Rocasolano". CSIC. Serrano, 199. 28006 Madrid.

(**) Universidade de Santiago de Compostela. Departamento de Historia 1. Facultade de Xeografía e Historia. 15704 Santiago de Compostela.

El artículo fue remitido en su versión final el 15-XII-98.

\begin{abstract}
The excavation of the hillfort of OAchadizo has brought to light several domestic structures intermingled with rubish heaps, belonging to the Iron A ge Castro culture of NW Iberia. The abundant organic remains have constituted the basis for a systematic programme of radiocarbon dating of the different stratigraphic levels, which shows that the main occupation of the site took place between the VI and the II centuries cal. BC. Moreover, we have checked the $14 C$ dates obtained from terrestrial samples (bones and charcoal) against those made on marine shells coming from the same strata. As a result, we have found that the latter have an apparent age of $316 \pm 19$ years, caused by the reservoir effect. Therefore, we stress the need for taking into account -and correcting whenever possible-such a deviation before using samples of marine origin for chronological purposes.
\end{abstract}

Palabras clave: Castro. Carbono 14. Fraccionamiento isotópico. Efecto reserva.

Key words: Iron Age hillfort. Radiocarbon. Isotopic fraccionation. Reservoir effect.

\section{INTRODUCCIÓN}

El castro de O Achadizo se localiza en una pequeña península sita en la margen septentrional de 
la ría de Arousa, a una altitud de $5 \mathrm{~m}$. sobre el nivel del mar, en un lugar ocupado hoy en día por el pueblo de Cabo de Cruz (Boiro, A Coruña) (Fig. 1). Los trabajos de apertura de una nueva calle en 1991 condujeron a la aparición de numerosos restos arqueológicos y al planteamiento de una excavación que se desarrolló entre dicho año y 1994, bajo la dirección de A. Concheiro Coello, abarcando un sector periférico del interior del asentamiento castreño, donde se descubrió una interesante serie de estructuras arquitectónicas, tanto domésticas como defensivas, yuxtapuestas a varios basureros. Estos últimos proporcionaron abundantes restos óseos, malacológicos e ictiológicos (Ferré et alii, 1996), además de un variado elenco artefactual, cuya distribución y características apuntaban a la existencia de una serie de ocupaciones correspondientes a posiblemente 4 fases sucesivas, extendiéndose desde la primera Edad del Hierro hasta el período galaico-romano.

La circunstancia, poco habitual en el Noroeste de la Península Ibérica, de disponer de una secuencia estratigráfica bastante prolongada junto con la existencia simultánea de abundantes restos orgánicos de naturaleza diferente (conchas de moluscos marinos, huesos y carbón vegetal) nos llevó a plantearnos un trabajo con vistas a alcanzar tres objetivos básicos: 1) obtener una serie de dataciones absolutas para el yacimiento mediante carbono $14 ; 2$ ) cotejar estos resultados con los materiales arqueológicos, a fin de situarlos en el tiempo o evaluar posibles discrepancias con la cronotipología; 3 ) calcular el efecto de la reserva marina de las conchas recogidas, en las que se sabe que existen proporciones anómalas de carbono 14 como se ha señalado en otros lugares de la costa atlántica peninsular (Cabral, 1990), con el objeto de poder calibrar sus fechas y compararlas con las de las muestras terrestres. De esos propósitos sólo el segundo se reveló impracticable, a pesar del obvio interés que ello tendría, a causa de la imposibilidad de obtener una relación exhaustiva y secuencial de los artefactos recuperados en este castro en tiempo y forma para completar la redacción de este artículo.

Por lo que respecta al interés de obtener una serie de fechas carbono 14 para el conjunto de las ocupaciones presentes en el castro de OAchadizo, creemos que resulta obvio, incluso si-lamentablemente-no podemos de momento confrontar los resultados radiométricos con la secuencia ergológica del yacimiento. La importancia de este esfuerzo radica, a nuestro entender, no sólo en dotar de una
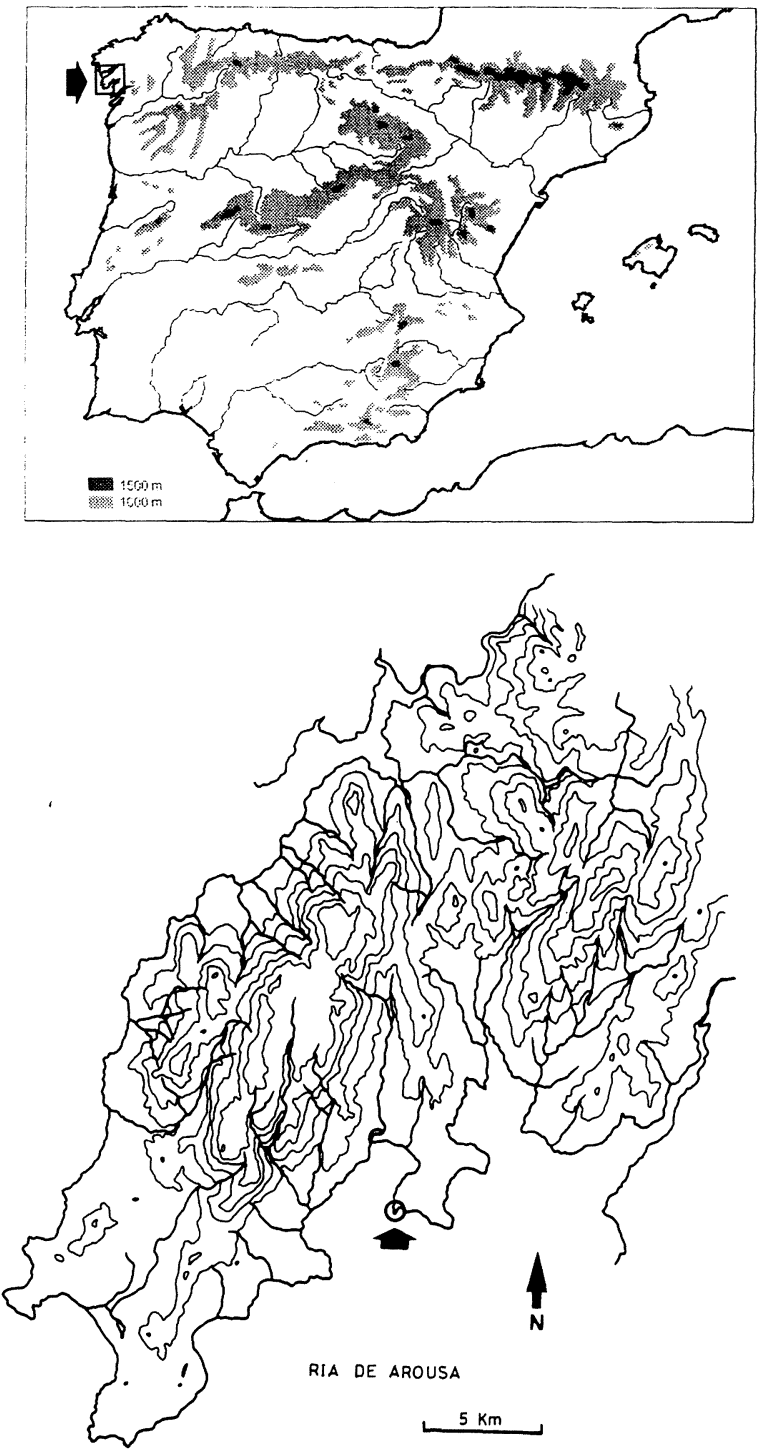

Fig. 1. Localización del castro de OAchadizo (Boiro, A Coruña).

cronología científicamente fundamentada a dicho poblado - una finalidad laudable per se-, sino en el carácter sistemático de esta labor, pues la veintena de dataciones disponibles nos permite soslayar el peligro de depender de unas pocas fechas aisladas, como tan a menudo sucede, y otorga además la posibilidad de localizar eventuales anomalías en algunio de los valores obtenidos, o incluso replantear en casos concretos las hipótesis sobre la constitución de alguna de las unidades del registro arqueológico. Un último factor que redunda en la necesidad de contar con el mayor número posible de fechas es la denominada "catástrofe de la Edad del 


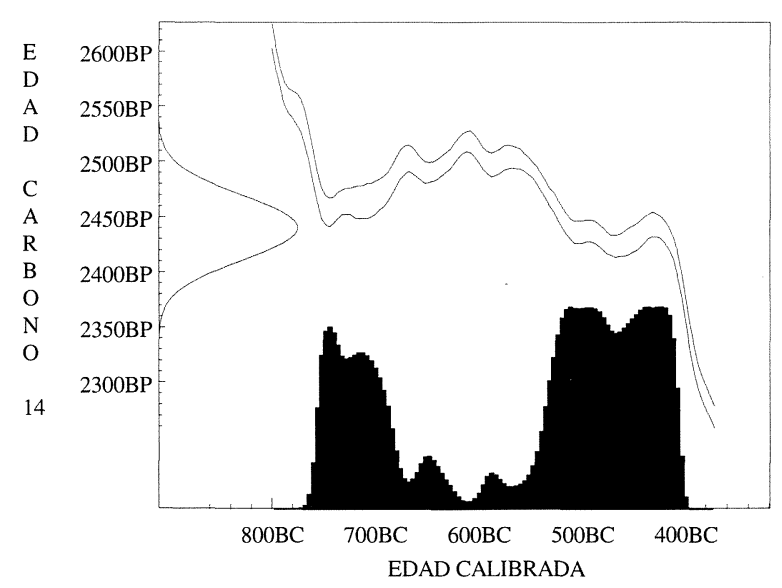

Fig. 2. Ejemplo de calibración con la muestra Utc-5660 de OAchadizo (Boiro, A Coruña). La edad carbono 14 convencional, $2440 \pm 29$ años BP, presenta una distribución simétrica alrededor del valor central en el eje de ordenadas. La curva de calibración (Stuiver et alii, 1993) relaciona la fecha convencional con la edad solar o calibrada. En este caso, presenta una meseta entre 800 y 400 cal BC que provoca que la distribución irregular de la edad calibrada (área en negro) se extienda en un intervalo de gran amplitud.

Hierro", expresión con la que se alude a un tramo amesetado en la curva de calibración entre 800 y 400 cal BC (1), lo que conlleva la aparición de intervalos calibrados desproporcionadamente amplios, incluso si como en la muestra C-1 de O Achadizo (UtC-5660) la desviación típica es relativamente pequeña (Fig. 2).

En cuanto a la pertinencia de recurrir al carbono 14 para la obtención de cronologías absolutas en momentos tan avanzados de la Prehistoria, hay autores que lo consideran innecesario, fiando más a las escasas referencias de autores clásicos o, sobre todo, a las informaciones proporcionadas por la tipología artefactual, especialmente los materiales de importación. Sin salirnos de nuestro ámbito de estudio, F. Calo (1997: 197, 208) se hace eco de esta posición en un reciente trabajo, glosando la inutilidad comparativa de los métodos radiométricos frente a la precisión de las secuencias tipológicas cerámicas, una opinión que nos parece poco fundamentada. En primer lugar, la exactitud de los análisis de carbono 14 puede incrementarse notablemente a través de una adecuada selección de las muestras, que incluye no sólo considerar su natu-

(1) Coincidente grosso modo con la primera Edad del Hierro y la transición entre ésta y la segunda fase de dicho período. Empleamos la terminología internacional en la que BC significa antes de Cristo. raleza y tamaño, sino también su relación concreta con el nivel o evento que se pretende datar, tareas que le corresponden al arqueólogo y que éste no siempre asume, por comodidad o ignorancia. Desde luego es necesario manejarse con series de fechas que luego se pueden evaluar individualmente, descartando aquellas con mayores desviaciones o simplemente anómalas, para a continuación combinarlas estadísticamente, aumentando así la fiabilidad del resultado. Por otra parte, los materiales cerámicos importados no son tan abundantes en la cultura castreña, salvo en su tramo final y a menudo la falta de adecuada publicación impide precisar las condiciones en que éstos han pasado a formar parte del registro arqueológico, reduciendo así, al menos en parte, su capacidad de proporcionar una cronología precisa. En realidad, las importantes limitaciones que aquejan todavía al conocimiento de los yacimientos castreños gallegos no aconsejan el prescindir sin más de una herramienta metodológica como es el carbono 14, que lógicamente ha de emplearse no de forma acrítica sino en combinación dialéctica con informaciones derivadas de la estratigrafía o de los análisis artefactuales. En otros lugares y culturas para los que se dispone de bastante más información así se está haciendo: valgan como ejemplo trabajos como el de Manning y Weninger (1992) correlacionando fechas carbono $14 \mathrm{e}$ históricas en el Heládico Reciente, o el de Hassan y Robinson (1987) en esa misma línea para el Egipto faraónico. En la propia Galicia y Norte de Portugal se han abordado en la década de los 90 trabajos de síntesis sobre el ya importante catálogo de dataciones radiométricas de procedencia castreña (Carballo y Fábregas, 1991) (2), cotejándolas también con las informaciones proporcionadas por el estudio de las cerámicas y otros materiales arqueológicos (Rey, 1996).

\section{CRONOLOGÍA}

En la excavación del yacimiento de OAchadizo se obtuvieron 20 muestras susceptibles de ser datadas por carbono 14, según se detalla en la tabla 1. Entre ellas había 7 muestras de carbón vegetal procedentes de hogares, 6 de huesos y 7 de conchas marinas, Patella vulgata en su mayoría. De estas

(2) En este apartado debemos citar igualmente la Tesis doctoral de F.M.V.R. Queiroga (1992): War and castros. New approaches to the northwestern Portuguese Iron Age, presentada en la Universidad de Oxford.

T. P., 56, n. ${ }^{\circ} 1,1999$ 
últimas, una de ellas (muestra K-2) presentaba dos tipos de conchas, lapas (Patella vulgata) y bígaros (Littorina littorea), por lo que se procesaron de forma independiente. La muestra C-1 de carbón no tenía cantidad suficiente para ser analizada según el procedimiento convencional, por lo que fue enviada al Van der Graaf Laboratorium de la Universidad de Utrecht (Holanda) para medirla medianteAMS (referencia UtC-5660). El resto de las muestras se realizaron en el Laboratorio de Geocronología del CSIC.

Las muestras de carbón se prepararon según el procedimiento ácido-álcali-ácido estándar (Mook y Waterbolk, 1985). En los huesos se obtuvo gelatina según el procedimiento de Longin (Longin, 1972). Las conchas fueron tratadas con $\mathrm{HCl} 2 \%$ hasta que su peso se redujera, al menos, en un $25 \%$, para evitar contaminaciones por recristalización (Olson y Broecker, 1961). La actividad del carbono 14 se midió, en función del peso final de la muestra, bien como dióxido de carbono en un contador proporcional, bien como benceno en un contador de centelleo líquido. Los resultados se muestran en la tabla 1.

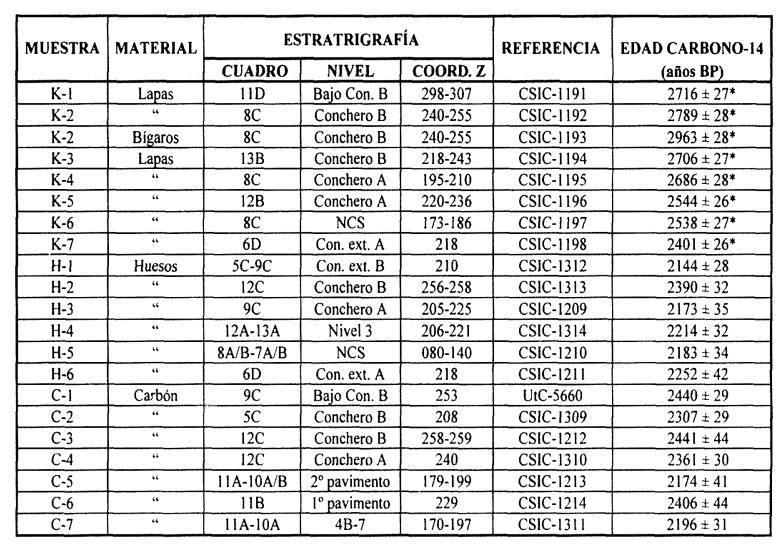

Tab. 1. Relación de muestras datadas por carbono 14 para O Achadizo (Boiro, A Coruña). (*) Para las conchas, las fechas no se pueden calibrar directamente y deben ser corregidas por el efecto de la reserva marina. Abreviaturas: NCS (nivel conchero superior); Con. (conchero); ext. (exterior).

\section{Problemática en la datación de conchas marinas}

A pesar de que muchas de las muestras de conchas de la tabla anterior son globalmente coetáneas con otras de carbón o de hueso, se observa como sus edades son sistemáticamente más antiguas. La causa de estas diferencias es conocida desde los inicios del método (Kulp et alii, 1951; Anderson y Libby, 1951; Rafter, 1955) y se debe a la menor concentración de carbono 14 en la reserva marina, causada tanto por el elevado tiempo de residencia del carbono en las capas profundas de los océanos como por la subida de éstas a zonas superficiales (upwelling), provocando con ello que el contenido en carbono 14 en la reserva marina no sólo sea menor sino también variable respecto al del medio terrestre.

El carbono 14 producido en los estratos superiores de la atmósfera se incorpora rápidamente en forma de $\mathrm{CO}_{2}$ a la biosfera terrestre y a la superficie del mar, pero una vez en el agua el intercambio con las capas más profundas es muy lento de tal manera que la actividad del carbono 14 en éstas es menor y proporciona edades más antiguas. Además, el sistema de intercambio no es estable sino que los niveles profundos ascienden periódicamente a'la superficie y se mezclan con las aguas superficiales, modificando su contenido en el isótopo. Este fenómeno, denominado upwelling, está causado por el efecto del viento y la rotación terrestre que expulsan las aguas superficiales hacia alta mar provocando la subida de aguas abisales. Las áreas más afectadas son las costas occidentales y las regiones polares, aunque en cada zona particular, el tipo de costa, el clima local, las corrientes y la clase de viento contribuyen a la variación en la magnitud del upwelling. La consecuencia de este fenómeno es que la concentración en carbono 14 de los carbonatos de las aguas superficiales disminuye, por lo que la actividad de éstos no es igual a la de las muestras terrestres. En general, esta diferencia se estima en aproximadamente 400 años, aunque debe cuantificarse en cada región al variar las condiciones que la generan.

El efecto de la reserva marina impide calibrar directamente las fechas de conchas. Para solucionar este problema se han seguido dos procedimientos. El primero consiste en determinar la edad aparente de las conchas, es decir, la divergencia temporal entre éstas y las muestras terrestres coetáneas. Una vez determinada la edad aparente media para el yacimiento, se puede restar del valor individual de cada concha y proceder a la calibración. Este método tiene como principal desventaja que no tiene en cuenta la variación en la concentración de carbono 14 con el tiempo debida al upwelling, por lo que sólo puede aplicarse al período concreto en el que se calcula. Por el contrario, permite corregir las edades

T. P., 56, n. ${ }^{\circ} 1,1999$ 
carbono 14 de las conchas y datar momentos sólo representados por éstas si no se alejan excesivamente en el tiempo. El segundo procedimiento para calibrar conchas se desarrolló en 1986 mediante la obtención de una curva de calibración específica para muestras marinas, que fue ampliada y mejorada en 1993 (Stuiver et alii, 1986; Stuiver y Braziunas, 1993), basadas ambas en el modelo global de difusión para el carbono descrito por Oeschger et alii (1975). Estas curvas determinan la edad solar de una muestra marina en función de su data carbono 14 convencional y de un parámetro, denominado $\Delta \mathrm{R}$, que cuantifica la desviación respecto al modelo debido al upwelling de cada zona. La curva de calibración lleva implícita la modificación temporal de la actividad carbono 14 con el tiempo y define un modelo de cómo cambia la reserva marina de forma global, mientras que $\Delta \mathrm{R}$ determina la variación peculiar de cada zona en función de sus condicionantes regionales y es, en primera aproximación, independiente del tiempo. Cuando $\Delta \mathrm{R}$ sea próximo a cero podremos concluir que el modelo ha dado una buena aproximación al comportamiento de la reserva marina en esa zona.

El cálculo de $\Delta \mathrm{R}$ sólo es posible a partir de la medida por carbono 14 de conchas cuya fecha de recolección sea conocida y anterior a 1950 d.C., para evitar la contaminación de los ensayos nucleares. También se puede realizar si se tienen muestras atmosféricas cuya edad se presupone semejante a la de las marinas (Dye, 1994), como es el caso de O Achadizo. Por último indicar que, aunque se ha descrito que $\Delta R$ es independiente del tiempo en primera aproximación, se han encontrado evidencias de variación de este valor asociado principalmente a cambios climáticos (3) o por aportes de aguas ricas en carbonatos inorgánicos de edad infinita procedentes de formaciones calcáreas (Heier-Nielsen et alii, 1995).

\section{Calibración de las fechas de O Achadizo}

En la tabla 2 se agrupan las muestras marinas y terrestres que se consideran coetáneas y que nos van a permitir contrastar los dos procedimientos de calibración en conchas. El primero radica en comparar las edades carbono-14 de las conchas con las de carbón o hueso para determinar la edad aparen-

(3) Documentadas por A.M. Soares en su Tesis doctoral inédita (1989): $O$ efeito de reservatório oceânico nas águas costeiras de Portugal continental. ICEN-LNETI, Lisboa.

\begin{tabular}{|c|c|c|c|c|}
\hline REFERENCIA & MATERIAL & $\begin{array}{l}\text { EDAD CARBONO-14 } \\
\text { (años BP) }\end{array}$ & $\begin{array}{c}\text { EDAD APARENTE } \\
\text { (años) }\end{array}$ & $\begin{array}{c}\Delta \mathrm{R} \\
\text { (años) }\end{array}$ \\
\hline UtC-5660 & Carbón & $2440 \pm 29$ & & \\
\hline CSIC-1191 & Lapas & $2716 \pm 27$ & $276 \pm 40$ & $-26 \pm 27$ \\
\hline CSIC-1212 & Carbón & $244 l \pm 44$ & & \\
\hline CSIC-1192 & Lapas & $2789 \pm 28$ & $348 \pm 52$ & $46 \pm 28$ \\
\hline CSIC-1193 & Bigaros & $2963 \pm 28$ & $522 \pm 52^{*}$ & $220 \pm 28^{*}$ \\
\hline CSIC-13I2 & Huesos & $2390 \pm 32$ & & \\
\hline CSIC-1194 & Lapas & $2706 \pm 27$ & $316 \pm 42$ & $-4 \pm 27$ \\
\hline CSIC- 1310 & Carbón & $2361 \pm 30$ & & \\
\hline CSIC-1195 & Lapas & $2686 \pm 28$ & $325 \pm 25$ & $-14 \pm 28$ \\
\hline CSIC-1314 & Huesos & $2214 \pm 32$ & & \\
\hline CSIC-1196 & Lapas & $2544 \pm 26$ & $330 \pm 41$ & $-23 \pm 26$ \\
\hline CSIC-12I1 & Huesos & $2252 \pm 42$ & & \\
\hline CSIC-1198 & Lapas & $2401 \pm 26$ & $149 \pm 49^{*}$ & $-183 \pm 26^{*}$ \\
\hline \multicolumn{3}{|c|}{ VALOR MEDIO PONDERADO } & $316 \pm 19$ & $-5 \pm 12$ \\
\hline
\end{tabular}

Tab. 2. Cálculo de la edad aparente y $\Delta \mathrm{R}$ para las conchas de OAchadizo (Boiro, A Coruña). (*) Los valores de edad aparente y $\Delta R$ obtenidos para las muestras CSIC-1193 y CSIC-1198 son estadísticamente diferentes del resto según el test $\chi^{2}$, por lo que no se utilizan para hallar la media ponderada.

te, $R(t)$, o diferencia en años entre las reservas marina y terrestre. El segundo procedimiento calcula el $\Delta \mathbf{R}$ correspondiente a cada una de las agrupaciones de fechas de forma bastante más complicada y haciendo uso de las curvas de calibración (Stuiver y Braziunas, 1993). Como puede observarse, los valores coinciden bastante bien y tienen poca dispersión, tanto en lo que concierne a la edad aparente como a $\Delta \mathrm{R}$, excepto en las muestras $\mathrm{K}-2$ (bígaros) y K-7 (CSIC-1193 y CSIC-1198) cuyos valores son estadísticamente diferentes del resto y, por tanto, no se han considerado. El valor medio ponderado de $\Delta \mathrm{R}$ es de $-5 \pm 12$ años, muy próximo a cero, con lo que podemos concluir que el modelo global predice con mucha similitud el comportamiento de la reserva marina para la zona de OAchadizo en el primer milenio BC.

Con el dato de $\Delta \mathrm{R}$ se puede ya calcular la edad calibrada de las conchas marinas. Para ello se utiliza el programa Oxcal versión 2.18 de la Universidad de Oxford (Ramsey, 1995), al igual que para las muestras terrestres. Los resultados pueden verse en la tabla 3, en la que se da el intervalo de edaỏ calibrada a 2 sigma. No se corrigen las fechas de las muestras CSIC-1193 y CSIC-1198, puesto que en el cálculo del valor medio ponderado de ÄR no se han utilizado sus datos y el error al determinar sus edades calibradas sería considerable.

La figura 3 muestra la distribución de probabilidad para cada una de la fechas calibradas a 2 sigma, representadas cronológicamente con un rango de edad que oscila en conjunto entre los años 750 y 100 cal BC.Además, se dibuja la çurva de la suma de probabilidades, que consiste en una com- 


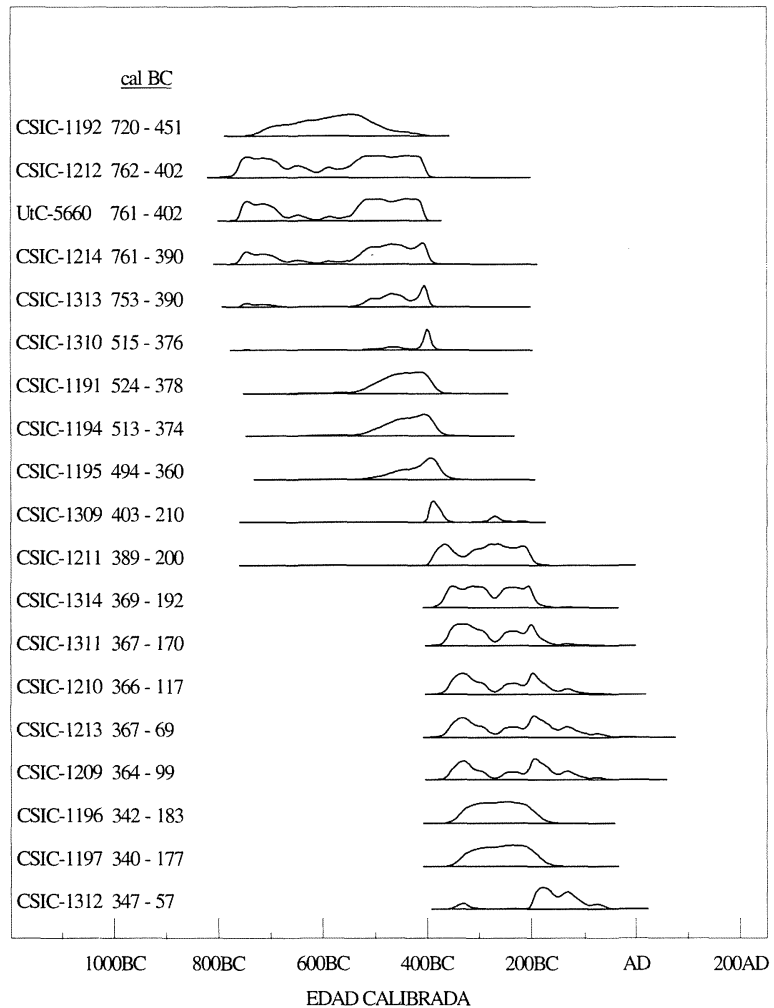

SUMA DE PROBABILIDADES

Intervalos: 755 - 681 cal BC $(5 \%)$

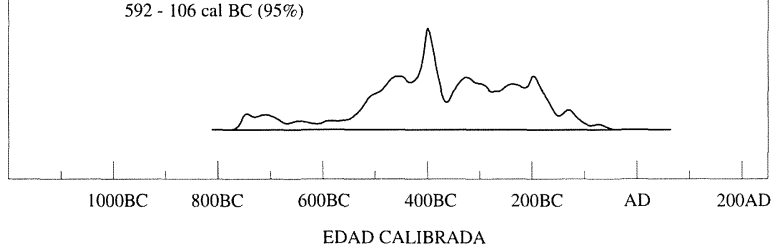

Fig. 3. Distribución y suma de probabilidades para las fechas carbono-14 de OAchadizo (Boiro, A Coruña).

binación de todas las fechas mediante la suma promediada de sus distribuciones de probabilidad. El intervalo obtenido debe interpretarse como el periodo en el que el $95 \%$ de los eventos tuvieron lugar y no como el periodo en el que todos los eventos sucedieron con una probabilidad del $95 \%$, que es diferente. El gráfico muestra así en qué momentos existe mayor probabilidad de que el yacimiento estuviera ocupado. Puede verse como se obtiene, para 2 sigma, un intervalo entre $755-681 \mathrm{cal} \mathrm{BC}$ con poca probabilidad de ocupación $(5 \%)$ y que el mayor peso (95\%) se lo lleva el intervalo 592 $106 \mathrm{cal} \mathrm{BC}$, con un pico destacable alrededor del 400 cal BC.

\begin{tabular}{|c|c|c|c|}
\hline MUESTRA & MATERIAL & $\begin{array}{l}\text { EDAD CALIBRADA } \\
\text { (años cal BC) }\end{array}$ & $\begin{array}{l}\text { COMBINACIÓN DE } \\
\text { PROBABILIDADES }\end{array}$ \\
\hline CSIC-1192 & Lapas & $720-451(1.00)$ & $\ldots$ \\
\hline CSIC-1212 & Carbón & $\begin{array}{l}762-625(0.38) \\
598-402(0.62) \\
\end{array}$ & \multirow{8}{*}{ cal BC 477 - 399} \\
\hline UtC- 5660 & “ & $\begin{array}{l}761-673(0.30) \\
663-634(0.03) \\
553-402(0.66)\end{array}$ & \\
\hline CSIC- 1214 & “ & $\begin{array}{l}761-672(0.23) \\
663-632(0.03) \\
663-632(0.01) \\
555-390(0.73)\end{array}$ & \\
\hline CSIC-1313 & Huesos & $\begin{array}{l}753-700(0.10) \\
527-390(0.90) \\
\end{array}$ & \\
\hline CSIC-1310 & Carbón & $515-376(1.00)$ & \\
\hline CSIC-1191 & Lapas & $524-378(1.00)$ & \\
\hline CSIC-1194 & . & $513-374(1.00)$ & \\
\hline CSIC-1195 & “ & $494-360(1.00)$ & \\
\hline CSIC-1309 & Carbón & $\begin{array}{l}403-356(0.76) \\
292-241(0.22) \\
225-210(0.02) \\
\end{array}$ & $\ldots$ \\
\hline CSIC-12II & Huesos & $\begin{array}{l}389-334(0.28) \\
329-200(0.72) \\
\end{array}$ & \multirow{8}{*}{ cal BC 342 - 193} \\
\hline CSIC-1314 & “ & $369-192(1.00)$ & \\
\hline CSIC-131I & Carbón & $367-170(1.00)$ & \\
\hline CSIC- 1210 & Huesos & $\begin{array}{l}366-275(0.48) \\
265-151(0.47) \\
146-117(0.05)\end{array}$ & \\
\hline CSIC-1213 & Carbón & $\begin{array}{r}367-273(0.39) \\
267-94(0.60) \\
80-69(0.01) \\
\end{array}$ & \\
\hline CSIC-1209 & Huesos & $\begin{array}{ll}364-277 & (0.40) \\
263-99 & (0.60) \\
\end{array}$ & \\
\hline CSIC-1196 & Lapas & $342-183(1.00)$ & \\
\hline CSIC-1197 & & $340-177(1.00)$ & \\
\hline CSIC-1312 & Huesos & $\begin{array}{l}347-316(0.07) \\
203-57 \quad(0.93) \\
\end{array}$ & $\cdots$ \\
\hline CSIC-1193 & Bigaros & $\ldots$ & $\ldots$ \\
\hline CSIC-1198 & Lapas & $\ldots$ & - \\
\hline
\end{tabular}

Tab. 3. Calibración y combinación de probabilidades de las fechas de OAchadizo (Boiro, A Coruña). Las muestras terrestres se corrigen con la curva bidecadal (Stuiver et alii, 1993) mientras que para las conchas se recurre a la curva marina (Stuiver y Braziunas, 1993), aplicando un $\Delta \mathrm{R}$ igual a $-5 \pm 12$. En ambos casos se proporcionan los intervalos obtenidos calibrando a 2 sigma y la probabilidad de cada uno de ellos entre paréntesis en tanto por uno. La combinación de probabilidades de las fechas se realiza sobre el valor de la edad calibrada. Para todos los cálculos se utiliza el programa Oxcal 2.18 de la Universidad de Oxford (Ramsey, 1995). Las muestras CSIC-1193 y CSIC-1198 no se calibran (ver texto).

\section{Interpretación de resultados}

El valor de $\Delta \mathrm{R}$ próximo a cero indica que el modelo establecido por Stuiver y Braziunas (1993) explica bien el contenido de carbono 14 existente en la costa de Boiro durante el primer milenio BC. Eso contrasta con los valores obtenidos en otras zonas de orografía semejante a las rías gallegas. Así, Heier-Nielsen et alii (1995) demostraron que para los fiordos daneses el monto de $\Delta \mathrm{R}$ pierde su sentido al existir una gran dispersión en los valores, que oscilan entre 82 y 554 años con una media de 279 años, una circunstancia que achacan tanto a que el estudio se realizó sobre varios fiordos (con la variabilidad de condiciones que esto introduce) como a la entrada de aguas de ríos con alto contenido en carbonatos inorgánicos procedentes de depósitos calcáreos de edad infinita. Por el contrario, la edad 
aparente obtenida es comparable a la cifra conseguida por Soares (ver nota 2) para la zona comprendida entre Aveiro y Cabo de San Vicente, de $360 \pm 35$ años. En su trabajo sobre conchas de la costa portuguesa, Soares obtiene dos valores de $\Delta \mathrm{R}$ a partir de conchas de edad conocida en función de la latitud pero que según él sólo son válidos entre 0 y $1100 \mathrm{BP}$, pues a partir de entonces un posible cambio climático originó una variación intensa del upwelling en dichas zonas haciendo inoperantes los cálculos de $\Delta \mathrm{R}$ efectuados. Por ello, y a partir de muestras arqueológicas coetáneas de conchas y materiales terrestres, fija el valor de edad aparente en $360 \pm 35$ años para utilizar a partir del 2000 BP y hasta el inicio del Holoceno, el cual es próximo a los $316 \pm 19$ años obtenidos en OAchadizo.

Por otro lado, hay que tratar de explicar la presencia de valores discordantes en el cálculo tanto de la edad aparente como de $\Delta \mathrm{R}$. Uno de ellos es la única muestra de conchas de bígaros (CSIC-1193) $\mathrm{y}$, aunque un dato solo no nos permita concluir nada, se puede comentar que parece apoyar las tesis de Lynn Ingram y Southon (1996), los cuales al estudiar moluscos de la bahía de San Francisco se encontraron con variabilidades importantes según el tipo de concha datada. Así, argumentaron que el hábitat, el tipo de alimento y el periodo anual de crecimiento de la concha eran factores que podían determinar la variación de sus contenidos en carbono $14, y$, por tanto, de la edad que éste proporcionaba. Respecto a la otra, CSIC-1198 del Conchero exterior A, lugar de donde fueron tomadas tanto ésta como la muestra supuestamente coetánea (CSIC-1211), presenta una estratigrafía confusa que pretendía ser aclarada, al menos parcialmente, por las dataciones radiométricas. Éstas parecen indicar que la asociación entre las dos muestras no fue correcta pues la diferencia en los valores de $\Delta \mathrm{R}$ así lo demuestra. En todo caso no resulta sorprendente que, dada la complejidad de los procesos deposicionales y postdeposicionales en yacimientos de naturaleza doméstica y/o ocupaciones prolongadas, cuando se lleva a cabo un programa sistemático de datación C-14 surjan siempre dataciones "extrañas" que nos indican precisamente la complicada génesis de ese tipo de depósitos, no siempre adecuadamente valorable mediante un simple sondeo y la ocasional fecha radiométrica (4).

(4) Dentro del Noroeste tenemos un buen ejemplo de este tipo de problemática en otro yacimiento, el abrigo de Buraco da Pala, objeto de un minucioso estudio radiométrico (Sanches et alii, 1993; Sanches, 1997).
En cuanto a la cronología, todas las muestras datadas se enmarcan dentro de la segunda mitad del primer milenio $\mathrm{BC}$, pudiéndose apreciar una ocupación continua entre 592-106 cal BC. Los momentos de mayor intensidad en este periodo pueden deducirse de la combinación de aquellas fechas estadísticamente semejantes, de tal manera que se consiga una probabilidad común que solape al menos un $60 \%$ con las distribuciones individuales (Ramsey, 1995). Esta técnica permite reducir el intervalo de calibración tomando aquellos lapsos comunes a una serie de fechas que cumplan el criterio mencionado. En este trabajo se consigue así soslayar el problema de la meseta de la curva de calibración al tener más peso estadístico los intervalos más modernos, como puede apreciarse en la tabla 3. Para realizar estos cálculos se utilizó de nuevo el programa Oxcal, que obtuvo los dos agrupamientos que se muestran en ella, quedando aisladas la fechas CSIC-1192, al comienzo de la serie, CSIC-1309 entre los dos primeros grupos y CSIC1312 al final.Así, el primer agrupamiento de fechas proporciona un intervalo, cal BC 477-399, que genera el pico más importante en el gráfico de la suma de probabilidades al ser muchos valores que ocupan un espacio temporal corto. El segundo agrupamiento, cal BC 342-193, distribuye su probabilidad en un tiempo mayor, por lo que presenta una meseta más uniforme. Como puede verse, los lapsos entre los distintos intervalos son pequeños, existiendo además entre los dos primeros una fecha, CSIC1309 , que cubre el periodo, lo que refuerza la posibilidad de una ocupación continuada durante la segunda mitad del primer milenio BC.

\section{CONCLUSIONES}

Yendo de lo general a lo particular, debemos comenzar por aludir al interés de detectar y evaluar los posibles sesgos que pueden existir en las determinaciones radiométricas efectuadas sobre material conquiliológico, de lo cual este trabajo no debe ser más que un primer paso. En efecto, es muy frecuente que en yacimientos castreños del área más o menos litoral se hallen concheros, sobre cuyas características y composición se ha investigado en los últimos años (v.g. Troncoso et alii, 1995-1996; Rodríguez y Fernández, 1996) y que obviamente poseen una abundante materia prima para realizar dataciones carbono 14 de forma sistemática. El campo de aplicación de esa clase de trabajos no se limita a los 
asentamientos de la Edad del Hierro en exclusiva, pues se tienen referencias sobre la presencia de esa clase de restos en un yacimiento anterior a éstos, el de Guidoiro Areoso (Vilanova deArousa, Pontevedra) cuya única datación se ha efectuado precisamente sobre una muestra de conchas, que ha servido para plantear la posibilidad de una temprana presencia en el Noroeste de la aleación $\mathrm{Cu} / \mathrm{Sn}$ (Comendador, 1995; Fernándezet alii, 1995). Esta hipótesis habrá de manejarse con precaución en tanto no se compruebe la inexistencia de desfases cronológicos iguales o superiores para conchas del III milenio que los que hemos constatado en este artículo para el I milenio en la misma ría.

En cuanto a la cuestión metodológica, hay que indicar que existe buena correlación entre las muestras terrestres y las marinas, lo que permite calcular el valor de $\Delta \mathrm{R}$ para este área y realizar la calibración de las muestras de conchas. Podemos señalar que, a pesar de la localización del castro de OAchadizo (y verosímilmente de los moluscos recolectados) en un área bastante interior de la ría de Arousa (por lo tanto relativamente influida por los aportes hídricos continentales), tanto la edad aparente de las conchas analizadas como el valor de $\Delta \mathrm{R}$ hallados se ajustan bastante a las estimaciones efectuadas en el medio oceánico. En este sentido, sería interesante contrastar los resultados de nuestro yacimiento con los obtenibles en puntos situados más hacia la boca de la ría o ya en mar abierto.

De la observación de la figura 3 se deduce que la principal ocupación del poblado tiene lugar en la segunda mitad del I milenio BC grosso modo, con una concentración de la probabilidad (¿efecto de una habitación más intensa?) en el período que va desde mediados del siglo V a comienzos del II BC, coincidente por otra parte con el auge habitacional en un buen número de castros del Noroeste (Carballo y Fábregas, 1991: 262).

Para finalizar, este trabajo pretende mostrar una metodología adecuada para la utilización de las fechas carbono 14 realizadas sobre conchas y señalar que no es posible usarlas sin determinar previamente la cuantía del efecto de la reserva marina en el área de estudio. Se hace necesario, pues, una asociación cuidadosa de muestras marinas y terrestres coetáneas a priori que permita efectuar el cálculo de $\Delta R$, lo cual se hace en muchos casos francamente difícil. Además, se ha intentado exponer cómo el uso de las herramientas estadísticas disponibles (suma de probabilidades, combinación de fechas, etc.) y una cantidad adecuada de fechas puede faci- litar la interpretación de éstas, sobre todo cuando presentan intervalos de calibración demasiado amplios para una correcta adscripción temporal.

\section{BIBLIOGRAFÍA}

Anderson, E.C. y Libby, W.F. (1951): "World-wide distribution of naturai radiocarbon". Physical Review, 81: 64-69.

CABraL, J.M.P. (1990): “Arqueometria no LNETI -Balanço e perspectivas". Arqueologia, 20: 110-123.

CAlo Lourido, F. (1997): "A síntese: o galaico-romano". En G. Pereira (coord.): Galicia fai dous mil anos. Ofeito diferencial galego, 1. Museo do Pobo Galego. Santiago de Compostela: 193-212.

Carballo Arceo, X. y Fábregas Valcarce, R. (1991): "Dataciones de carbono-14 para castros del noroeste peninsular".Archivo Español deArqueología, 64: 243264.

Comendador Rey, B. (1995): "Caracterización de la metalurgia inicial gallega: una revisión”. Trabajos de Prehistoria, 52(2): 111-129.

DYE, T. (1994): “Apparent ages of marine shells: Implications for archaeological dating in Hawai 'i". Radiocarbon, 36(1): 51-58.

FERnández Miranda, M.; Montero Ruiz, I. y Rovira LloRENS, S. (1995): "Los primeros objetos de bronce en el occidente de Europa”. Trabajos de Prehistoria, 52(1): 57-69.

Ferré, M.C.; Rey, J.M.; Concheiro, A. y VÁzquez, J.M. (1996): "Contribución al conocimiento ictiológico del Castro de "OAchadizo" (Cabo de Cruz, Coruña, Galicia)”. En P. Ramil; C. Fernández y M. Rodríguez (eds.): Biogeografía Pleistocena-Holocena de la Península Ibérica. Santiago de Compostela: 291-296.

Hassan, F.A. y Robinson, S.W. (1987): "High-precision radiocarbon chronometry of ancient Egypt, and comparisons with Nubia, Palestine and Mesopotamia". Antiquity, 61: 119-135.

Heier-Nielsen, S.; Heinemeier, J.; Nielsen, H.L. y Rud, N. (1995): "Recent reservoir ages for danish fjords and marine waters". Radiocarbon, 37(3): 875-882.

KulP, J.L.; FreEly, H.W. y Tryon, L.E. (1951): "Lamont natural radiocarbon measurements, I". Science, 114: 565-568.

LONGIN, R. (1971): “New method of collagen extraction for radiocarbon dating". Nature, 230: 241-242.

LyNn INGRAM, B. y SOUTHON, J.R. (1996): "Reservoir ages in Eastern Pacific coastal and estuarine waters". Radiocarbon, 38(3): 573-582.

Manning, S.W. y Weninger, B. (1992): "A light in the dark: archaeological wiggle matching and the absolute chronology of the close of the Aegean Late Bronze Age". Antiquity, 66: 636-663. 
MoOK, W.G. y Waterbolk, H.T. (1985): Radiocarbon dating. Handbooks for Archaeologists, 3. European Science Fundation, Strasbourg.

Oeschger, H.; Siegenthaler, U.: Schotterer, U. y GugelMANN, A. (1975): "A box diffusion model to study the carbon dioxide exchange in nature". Tellus, 27: 168192.

Olson, E.A. y Broecker, W.S. (1961): "Lamont natural radiocarbon measurements, VII". Radiocarbon, 3: 141175.

RAFTER, T.A. (1955): " ${ }^{14} \mathrm{C}$ variations in nature and the effect on radiocarbon dating". New Zealand Journal of Science and Technology B36: 363-369.

RAMSEY, C.B. (1995): "Radiocarbon calibration and analysis of stratigraphy: The Oxcal program". Radiocarbon, 37(2): 425-430.

Rey Castiñeira, P. (1996): "Referencias de tempo na cultura material dos castros galegos". En J.M. Hidalgo Cuñarro (coord.): A cultura castrexa galega a debate. Instituto de Estudios Tudenses. Tui: 159-206.

RODRÍGUEZ, C. y FERNÁNDEZ, C. (1996): "Una aproximación al estudio de los yacimientos castreños del litoral galaico: dimensiones ambientales y económicas”. En P.
Ramil; C. Fernández y M. Rodríguez (eds.): Biogeografía Pleistocena-Holocena de la Península Ibérica. Santiago de Compostela: 363-375.

Sanches, M. de J. (1997): Pré-história recente de Trás-osMontes e Alto Douro. Sociedade Portuguesa de Antropologia e Etnologia. Oporto.

Sanches, M. De J.; Soares, A.M. y Alonso Matthias, F. (1993): "Buraco da Pala (Mirandela): datas de radiocarbono e seu poder de resolução. Algumas reflexões". $1^{\circ}$ Congresso de Arqueologia Peninsular (Actas). Trabalhos de Antropologia e Etnologia, 33(1-2): 223-243.

Stuiver, M. y Braziunas, T.F. (1993): "Modelling atmospheric ${ }^{14} \mathrm{C}$ influences and ${ }^{14} \mathrm{C}$ ages of marine samples to 10,000 BC". Radiocarbon, 35(1): 137-190.

Stuiver, M.; Long, A. y KRA, R.S. (eds.) (1993): Special calibration issue. Radiocarbon 35(1). New Haven.

Stuiver, M.; Pearson, G.W. yBraziunas, T.F. (1986): "Radiocarbon age calibration of marine samples back to 9000 cal years BP". Radiocarbon, 28(2B): 980-1021.

TRONCOSO, J.S.; VÁZQuez, J.M. y URGORRI, V. (1995-1996): "La malacofauna en la alimentación de la cultura castreña de la Edad del Hierro en Galicia (NO de España)". Brigantium, 9: 107-114.

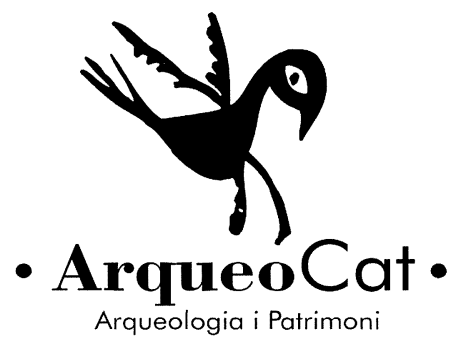

ARQUEOCAT S.L., DENTRO DE SUS SERVICIOS AL PATRIMONIO, LE OFRECE SU

SERVICIO DE LIBRERÍA

DIRIGIDO A TODOS LOS PROFESIONALES E INSTITUCIONES RELACIONADOS CON LA ARQUEOLOGÍA Y EL PATRIMONIO

LIBRO NACIONAL Y EXTRANJERO. BÚSQUEDA DE CUALQUIER PUBLICACIÓN QUE NO APAREZCA EN NUESTROS CATÁLOGOS.

SI DESEA MÁS INFORMACIÓN O RECIBIR NUESTROS CATÁLOGOS:

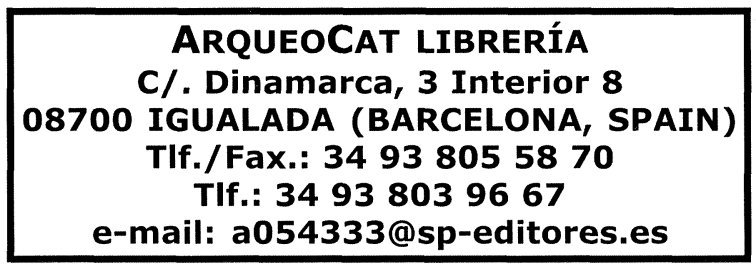

